Bull. Austral. Math. Soc.

VOL. 47 (1993) [291-296]

\title{
ON SKEW-COMMUTING MAPPINGS OF RINGS
}

\author{
Matej BREšar
}

\begin{abstract}
A mapping $f$ of a ring $R$ into itself is called skew-commuting on a subset $S$ of $R$ if $f(s) s+s f(s)=0$ for all $s \in S$. We prove two theorems which show that under rather mild assumptions a nonzero additive mapping cannot have this property. The first theorem asserts that if $R$ is a prime ring of characteristic not 2, and $f: R \rightarrow R$ is an additive mapping which is skew-commuting on an ideal $I$ of $R$, then $f(I)=0$. The second theorem states that zero is the only additive mapping which is skew-commuting on a 2-torsion free semiprime ring.
\end{abstract}

Let $S$ be a subset of a ring $R$. A mapping $f$ of $R$ into itself is said to be skewcommuting on $S$ if $f(s) s+s f(s)=0$ for all $s \in S$. For results on skew-commuting mappings and their generalisations (such as semi-commuting, skew-centralising, semicentralising mappings) we refer the reader to $[4,6,7,8]$. In these papers the authors have showed that nonzero derivations and ring endomorphisms cannot be skewcommuting (semi-commuting, ...) on certain subsets of prime rings (for example, ideals). In the present paper we prove theorems of this kind for general additive mappings. Our first result is

THEOREM 1. Let $R$ be a prime ring of characteristic not 2. If an additive mapping $f: R \rightarrow R$ is skew-commuting on some ideal $I$ of $R$, then $f(x)=0$ for all $x \in I$.

Clearly, the requirement that the characteristic of $R$ is not 2 cannot be removed (consider, for instance, the identity on $R$ ). In fact, if the characteristic of a ring $R$ is 2 , then the notion of skew-commuting mappings coincides with the notion of commuting mappings, that is, the mappings $f$ satisfying $f(x) x=x f(x)$. In [1] we showed that every additive commuting mapping of a prime ring $R$ (of arbitrary characteristic) is of the form $x \rightarrow \lambda x+\zeta(x)$ where $\lambda$ is an element in $C$, the extended centroid of $R$, and $\zeta$ is an additive mapping of $R$ into $C$ (see also $[2,3]$ for similar results). The fact that the structure of commuting mappings can be described has been one of the main motivations for this research.

\section{Received 2 April 1992}

This work was supported by the Research Council of Slovenia.

Copyright Clearance Centre, Inc. Serial-fee code: 0004-9729/93 \$A2.00+0.00. 
Suppose a ring $R$ contains nonzero ideals $I$ and $J$ such that $I J=0=J I$ (thus $R$ is not prime). Any mapping $f$ of $R$ with range contained in $J$ is certainly skewcommuting on $I$; however, it does not necessarily vanish on $I$. Thus Theorem 1 does not hold for semiprime rings in general. Nevertheless, the following is true:

TheOREM 2. Let $R$ be a 2-torsion free semiprime ring. If an additive mapping $f: R \rightarrow R$ is skew-commuting on $R$, then $f=0$.

Theorem 2 will follow easily from Theorem 1. In order to prove Theorem 1 we define $I_{n}=\left\{x^{n} \mid x \in I\right\}$ ( $n$ is a positive integer), and let us prove

Lemma 1. Let $R$ be a prime ring, $I$ be a nonzero ideal of $R$, and $a \in R$. If there exists a positive integer $n$ such that $I_{n} a=0$ (or $a I_{n}=0$ ), then $a=0$.

Proof: Suppose $a \neq 0$. Since $R$ is prime there exists $w \in I$ such that $a w \neq 0$. For any $x \in R$, the element $a w x$ lies in $I$, hence $(a w x)^{n} a=0$ for all $x \in R$. But then $(a w x)^{n+1}=0, x \in R$, and so [5, Lemma 1.1] yields $a w=0$, contrary to the assumption. Similarly one discusses the case when $a I_{n}=0$.

Proof of Theorem 1: For the proof we need several steps. We begin with

Lemma A. For $x, y \in I$,

(1) $f(x) y+y f(x)+f(y) x+x f(y)=0$ for all $x, y \in I$.

(2) $x^{4} f(x)=0=f(x) x^{4}$.

Proof: Linearising $f(x) x+x f(x)=0$ we obtain (1). Let us prove (2). From the initial hypothesis we see that for any $x \in I, f(x)$ commutes with $x^{2}$. Therefore, replacing $y$ by $x^{2}$ in (1) we obtain

$$
2 x^{2} f(x)+f\left(x^{2}\right) x+x f\left(x^{2}\right)=0 \text { for all } x \in I .
$$

Multiply (3) from the right by $x^{2}$; since $f(x) x^{2}=x^{2} f(x)$ and since, by the initial hypothesis, $f\left(x^{2}\right) x^{2}+x^{2} f\left(x^{2}\right)=0$, it follows that

$$
2 x^{4} f(x)=x^{2} f\left(x^{2}\right) x+x^{3} f\left(x^{2}\right) .
$$

On the other hand, by (3) we see that

$$
2 x^{4} f(x)=x^{2}\left(2 x^{2} f(x)\right)=-x^{2} f\left(x^{2}\right) x-x^{3} f\left(x^{2}\right)
$$

Comparing the last two relations we arrive at $4 x^{4} f(x)=0$. We have assumed that the characteristic of $R$ is not 2 , and so $x^{4} f(x)=0$. Since $f(x) x=-x f(x)$, we also have $f(x) x^{4}=0$. 
Lemma B. For $u \in I_{10}, y \in I, u f(y) u=0$.

Proof: Multiply (1) from the left and from the right by $x^{4}$. According to (2) we obtain

$$
x^{4} f(y) x^{5}+x^{5} f(y) x^{4}=0 \text { for all } x, y \in I .
$$

Taking $x^{2}$ for $x$ in (4) we get

$$
x^{8} f(y) x^{10}+x^{10} f(y) x^{8}=0 .
$$

But from (4) if follows that

$$
\begin{aligned}
x^{8} f(y) x^{10} & =x^{4}\left(x^{4} f(y) x^{5}\right) x^{5} \\
& =-x^{4}\left(x^{5} f(y) x^{4}\right) x^{5} \\
& =-x^{5}\left(x^{4} f(y) x^{5}\right) x^{4} \\
& =x^{5}\left(x^{5} f(y) x^{4}\right) x^{4} \\
& =x^{10} f(y) x^{8} .
\end{aligned}
$$

Comparing the last two identities one concludes that $x^{8} f(y) x^{10}=0$ for all $x, y \in I$. But then also $x^{10} f(y) x^{10}=0$, which is the assertion of the lemma.

There is nothing to prove if $I=0$. Therefore, we assume henceforth that $I \neq 0$.

Lemma C. There exists a nonzero left ideal $L$ of $R$, contained in $I$, such that $f(L)=0$.

Proof: As a special case of (1) we have

$$
f(x) u+u f(x)+f(u) x+x f(u)=0 \text { for all } x \in I, u \in I_{10} .
$$

Multiplying (5) from the right by $u$, and then using Lemma $B$, we arrive at

$$
f(x) u^{2}+f(u) x u+x f(u) u=0 \text { for all } x \in I, u \in I_{10} .
$$

Suppose $x \in I_{10}$. By Lemma $B$ we then see that $x f(u) x=0$, and also $x^{2} f(x)=$ $-x f(x) x=0$. Therefore it follows from (6) that $x^{3} f(u) u=0$. That is, $v f(u) u=0$ for all $v \in I_{30}, u \in I_{10}$. By Lemma 1 we then have $f(u) u=0$. Thus (6) reduces to

$$
f(x) u^{2}+f(u) x u=0 \text { for all } x \in I, u \in I_{10} .
$$

Substituting $x u$ for $x$ in (7) we obtain $f(x u) u^{2}+f(u) x u^{2}=0$. On the other hand, $f(u) x u^{2}=(f(u) x u) u=-f(x) u^{3}$. Consequently we have

$$
f(x u) u^{2}=f(x) u^{3} \text { for all } x \in I, u \in I_{10} .
$$


Now, multiply (5) from the left by $u$. Since $u f(x) u=0$ and $u f(u)=-f(u) u=0$, it follows that $u^{2} f(x)+u x f(u)=0, x \in I, u \in I_{10}$. Replacing $x$ by $x u$ in this relation, and applying $u f(u)=0$, we then get

$$
u^{2} f(x u)=0 \text { for all } x \in I, u \in I_{10} .
$$

As a special case of (1) we have

$$
f(x) y u+y u f(x)+f(y u) x+x f(y u)=0
$$

for all $x, y \in I, u \in I_{10}$. Multiply this identity from the left and from the right by $u^{2}$. In view of Lemma $B,(9)$ and (8), we then get $u^{2} f(x) y u^{3}+u^{2} x f(y) u^{3}=0$. Hence

$$
v f(x) y v+v x f(y) v=0
$$

holds for all $v \in I_{30}, x, y \in I$. Replace in this relation $y$ by $y v f(z)$ where $y, z \in I$, $v \in I_{30}$. Then the first term is zero by Lemma B, so we have $v x f(y v f(z)) v=0$. Since $R$ prime it follows that

$$
f(y v f(z)) v=0 \text { for all } y, z \in I, v \in I_{30} .
$$

Substituting $y v f(z)$ for $y$ in (1) we obtain

$$
f(x) y v f(z)+y v f(z) f(x)+f(y v f(z)) x+x f(y v f(z))=0 .
$$

Multiplying from the right by $v$, and using Lemma B and (10), we then obtain

$$
y v f(z) f(x) v+f(y v f(z)) x v=0 \text { for all } x, y, z \in I, v \in I_{30} .
$$

Taking $r y$ for $y$, where $r \in R$ and $y \in I$, we get

$$
\operatorname{ryv} f(z) f(x) v+f(\operatorname{ryv} f(z)) x v=0 .
$$

On the other hand we see from (11) that

$$
\operatorname{ryv} f(z) f(x) v=-r f(y v f(z)) x v .
$$

Comparing we obtain

$$
\{f(r y v f(z))-r f(y v f(z))\} x v=0
$$

for all $r \in R, x, y, z \in I, v \in I_{30}$. The primeness of $R$ yields

$$
f(r y v f(z))=r f(y v f(z)) \text { for all } r \in R, y, z \in I, v \in I_{30} .
$$


Multiply (12) from the left and from the right by $u \in I_{10}$. In view of Lemma $B$ it follows that $u r f(y v(z)) u=0$. Thus $f(y v f(z)) u=0$, and so, by Lemma 1 ,

$$
f(y v f(z))=0 \text { for all } y, z \in I, v \in I_{30} .
$$

We may assume that $f(z) \neq 0$ for some $z \in I$. By Lemma $1, v f(z) \neq 0$ for some $v \in I_{30}$. Hence $a=x v f(z) \neq 0$ for some $x \in I$. Thus $L=R a$ is a nonzero left ideal of $R$, and since $a \in I, L$ is contained in $I$. By (13), $f(L)=0$.

Lemma D. $f(I)=0$.

Proof: From $f(L)=0$ and (1) it follows at once that

$$
f(x) t+t f(x)=0 \text { for all } t \in L, x \in I .
$$

Replacing $t$ by $r t$, where $r \in R$ and $t \in L$, it follows that $f(x) r t+r t f(x)=0$. By (14), the second term is equal to $-r f(x) t$, therefore $(f(x) r-r f(x)) t=0$ for all $r \in R$, $x \in I, t \in L$. Since $R$ is prime we then have $f(x) r-r f(x)=0$ for all $r \in R, x \in I$. That is, $f(x)$ lies in the centre of $R$ for every $x$ in $I$. But then (14) implies that $f(x) L=0, x \in I$, and therefore $f(x)=0$. With this the theorem is proved.

Proof of THEOREM 2: Since $R$ is semiprime, the intersection of all prime ideals in $R$ is zero.

Now pick a prime ideal $P$ such that $R / P$ is of characteristic not 2 . We want to show that $P$ is invariant under $f$. A linearisation of $f(x) x+x f(x)=0$ gives $f(x) y+y f(x)+f(y) x+x f(y)=0, x, y \in R$. Hence we see that

$$
f(p) x+x f(p) \in P \text { for all } p \in P, x \in R \text {. }
$$

In particular, $f(p) x y+x y f(p) \in P$ for all $p \in P, x, y \in R$. That is, $(f(p) x+x f(p)) y+$ $x(y f(p)-f(p) y) \in P$. The first term is contained in $P$ by (15), hence $x(y f(p)-f(p) y)$ $\in P, p \in P, x, y \in R$. Since $P$ is a prime ideal it follows that $y f(p)-f(p) y \in P$ for all $p \in P, y \in R$. Combining this statement with (15) we obtain $2 f(p) x \in P$. Since the characteristic of $R / P$ is not 2 it follows that $f(p) x \in P$ for all $p \in P, x \in R$. The ideal $P$ is prime, therefore, $f(p) \in P$ for every $p \in P$.

Since $f(P) \subseteq P, f$ induces an additive mapping $F$ on $R / P$, defined by $F(x+P)=f(x)+P$. Of course, $F$ is skew-commuting. Hence $F=0$ by Theorem 1.

Thus we have proved that the range of $f$ is contained in any prime ideal $P$ such that $R / P$ is of characteristic not 2 . The theorem will be proved by showing that the intersection of all such ideals is equal to zero. There exist prime ideals $\left\{P_{a} \mid a \in A\right\}$ such that $\bigcap_{a} P_{a}=0$. Let $B=\left\{b \in A \mid\right.$ the characteristic of $R / P_{b}$ is not 2$\}$ and $C=\left\{c \in A \mid\right.$ the characteristic of $R / P_{c}$ is 2$\}$. Thus $2 x \in \bigcap_{c} P_{c}$ for every $x \in R$. Therefore, given $x \in \bigcap_{b} P_{b}$, we have $2 x \in\left(\bigcap_{c} P_{c}\right) \cap\left(\bigcap_{b} P_{b}\right)=\bigcap_{a} P_{a}=0$, and so $x=0$ since $R$ is 2 -torsion free. Thus $\bigcap_{b} P_{b}=0$. 
REMARK. A mapping $f$ of a ring $R$ is called semi-commuting on a subset $S$ of $R$ if for any $x \in S$, either $f(x) x+x f(x)=0$ or $f(x) x-x f(x)=0$. Suppose that $R$ is 2-torsion free and 3-torsion free, and suppose that $f$ is an additive mapping of $R$ which is semi-commuting on some additive subgroup $S$ of $R$. We claim that in this case $f$ is either commuting on $S$ or skew-commuting on $S$. Indeed, introducing biadditive mappings $A: S \times S \rightarrow R$ and $B: S \times S \rightarrow R$ by $A(x, y)=f(x) y+x f(y)$ and $B(x, y)=f(x) y-x f(y)$, we have $S=P \cup Q$ where $P=\{x \in S \mid A(x, x)=$ $0\}, Q=\{x \in S \mid B(x, x)=0\}$. Suppose our assertion is not true, thus $P \neq S$ and $Q \neq S$. This means that $A(x, x) \neq 0$ and $B(y, y) \neq 0$ for some $x, y \in S$. Then, of course, $A(y, y)=0$ and $B(x, x)=0$. Now, consider the element $x+y$. If $x+y \in P$ then we have $A(x, x)+A(x, y)+A(y, x)=0$, and if $x+y \in Q$ then $B(x, y)+B(y, x)+B(y, y)=0$. Similarly we consider the elements $x-y$ and $x+2 y$. But then one can easily see that (since $R$ is 2-torsion free and 3-torsion free) either $A(x, x)=0$ or $B(y, y)=0$, contrary to the assumption. This proves our assertion. According to Theorem 1 we then obtain the following result: Let $f$ be an additive mapping of a prime ring of characteristic not 3 . If $f$ is semi-commuting on some ideal $I$ of $R$, then $f$ is commuting on $I$. Note that this result fairly generalises a theorem in [4].

\section{REFERENCES}

[1] M. Brešar, 'Centralizing mappings and derivations in prime rings', J. Algebra (to appear).

[2] M. Brešar, 'Centralizing mappings on von Neumann algebras', Proc. Amer. Math. Soc. 111 (1991), 501-510.

[3] M. Brešar, W.S. Martindale and C.R. Miers, 'Centralizing maps in prime rings with involution', J. Algebra (to appear).

[4] L.O. Chung and J. Luh, 'On semicommuting automorphisms of rings', Canad. Math. Bull. 21 (1978), 13-16.

[5] I.N. Herstein, Topics in ring theory (University of Chicago Press, Chicago, 1969).

[6] Y. Hirano, A. Kaya and H. Tominaga, 'On a theorem of Mayne', Math. J. Okayama Univ. 25 (1983), 125-132.

[7] A. Kaya, 'A theorem on semi-centralizing derivations of prime rings', Math. J. Okayama Univ. 27 (1985), 11-12.

[8] A. Kaya and C. Koc, 'Semicentralizing automorphisms of prime rings', Acta Math. Acad. Sci. Hungar. 38 (1981), 53-55.

University of Maribor

PF, Koros̄ka 160

62000 Maribor

Slovenia 